Case report

\title{
Cerebral Palsy and Heterotaxy Syndrome: A Case Report
}

\author{
Abba Musa Abdullahi ${ }^{1}{ }^{*}\left(\mathbb{C}\right.$, Ibrahim Muhammad Abdullahi ${ }^{2}$ \\ ${ }^{1}$ Life Sciences, University of South Wales, Newport, UK \\ ${ }^{2}$ Biomedical Sciences, University of Nottingham, Nottingham, UK \\ *Correspondence: amusabdullahi48@gmail.com
}

How to cite this paper: Abdullahi,

A. M., \& Abdullahi, I. M. (2021).

Cerebral Palsy and Heterotaxy

Syndrome: A Case Report. Global

Journal of Medical Case Reports, 1(1),

13-18. Retrieved from

https://www.scipublications.com/jou

rnal/index.php/gjmcr/article/view/17 2

Received: September 30, 2021

Accepted: November 4, 2021

Published: November 5, 2021

Copyright:@ 2021by the authors. Submitted for possible open access publication under the terms and conditions of the Creative Commons Attribution (CC BY) license (http://creativecommons.org/licenses /by/4.0/).

\begin{abstract}
Background: Cerebral palsy is not only a serious neurodevelopmental disease causing significant morbidity in children, but also a traumatic experience leading to psychosocial trauma to the parents/caregivers of the affected children. It is usually caused by prenatal or early post-natal insults to the newborn brain which may be associated with some congenital syndromes like congenital heart disease with transposition of the viscera but rarely a heterotaxy syndrome, a condition characterized with congenitally abnormal arrangement of the thoracic and abdominal viscera. Method: We present a case report of a 12-month-old boy with neurodevelopmental delay, recurrent episodes of non-mucoid and non-bloody diarhoea, occasional constipation, bilious vomiting, abdominal distension and fever with associated cough and difficulty in breathing. Results: We discuss an unusual presentation of cerebral palsy and heterotaxy syndrome diagnosed clinically with supporting evidence from both laboratory and radiological tests. Cerebral palsy was diagnosed from the history of birth asphyxia, delayed developmental milestone, limb spasticity and low values for all sub-scores of Bayley-III scale. Heterotaxy syndrome was diagnosed from the radiologic evidence of dextrocardia, left-sided stomach, centrally located liver and malrotation of gut with volvulus. We also provide a brief literature review of the incidence and prevalence, causes and risk factors, classification, clinical presentation and associated co-morbidities of heterotaxy syndrome. Conclusion: Diagnosis of heterotaxy syndrome in a child with background cerebral palsy is a great challenge to both physicians and radiologists. This is more so in developing countries due to poor availability of good diagnostic apparatus, therefore, a high index of suspicion is needed. A clear understanding of the clinical features, comprehensive history taking and thorough physical examination are important in making prompt diagnosis. Timely and appropriate imaging is necessary to prevent delays in diagnosis and treatment which lead to poor outcomes.
\end{abstract}

Keywords: Cerebral Palsy, Heterotaxy Syndrome, Congenital, Malrotation, Dextrocardia

\section{Introduction}

Cerebral palsy $(\mathrm{CP})$ is a syndrome that is caused by congenital or acquired insults that occurs to the developing brain usually before birth or during the early stage of infancy resulting in motor impairment, abnormalities of communication, intellectual ability, and epilepsy[1]. CP was defined by The International Executive Committee for the Definition of Cerebral Palsy as a group of disorders of the development of movement and posture, causing activity limitations which are attributed to non-progressive disturbances that occurred in the developing fetal or infant brain. The motor disorders of cerebral palsy are frequently accompanied by disturbances of sensation, cognition, communication, perception and/or behavior, and/or by a seizure disorder[2].

The term heterotaxy was derived from the Greek words: hetero and taxy. The former means 'other than' and the later means 'arrangement', that is 'other than normal arrangement'. Therefore, heterotaxy simply means a pattern of anatomical organization of the thoracic and the abdominal organs which is not the expected usual or normal 
arrangement [3]. Normally, the internal organs of the human body are organized into different patterns on both right and left sides of the body and are not mirror images of each other. Any symmetry or abnormal arrangement of thoracic or abdominal viscera can be called a 'heterotaxy' which also comprises of patients with a wide variety of very complex cardiac and extracardiac lesions[4]. Heterotaxy occurs as a result of failure of the developing embryo to establish normal left-right asymmetry that manifest typically as abnormal symmetry and malposition of the thoraco-abdominal viscera, complex congenital heart disease and extracardiac defects involving midline-associated structures[5]. Heterotaxy syndrome (HS) can be classified based on the presence or absence of a spleen as asplenia syndrome and polysplenia syndrome; or based on the architecture of the thoraco-abdominal viscera as heterotaxy with isomerism of the right atrial appendages and heterotaxy with isomerism of the left atrial appendages[6].

The term 'heterotaxy' is defined by the Nomenclature Working Group as "Heterotaxy is synonymous with 'visceral heterotaxy' and 'heterotaxy syndrome'. Heterotaxy is defined as an abnormality where the internal thoraco-abdominal organs demonstrate abnormal arrangement across the left-right axis of the body. By convention, heterotaxy does not include patients with either the expected usual or normal arrangement of the internal organs along the left-right axis, also known as 'situs solitus', nor patients with complete mirror-imaged arrangement of the internal organs along the left-right axis also known as 'situs inversus'." [7]. HS can be associated with many cardiac and extracardiac abnormalities involving lungs, liver, spleen, intestine, central nervous system and other midline defects. However, neurodevelopmental abnormalities were scarcely reported in association with HS and, to our knowledge, no extensive report of HS associated with cerebral palsy. Therefore, the aim of this report is to describe a 12 months old neonate with HS associated with cerebral palsy and to review existing literature on HS and the associated abnormalities.

\section{Case Presentation}

A 12 months old infant presented with recurrent episodes of non-mucoid and non-bloody diarhoea with occasional constipation; bilious vomiting; abdominal distension and fever for 4 weeks with left ear discharge for 2 weeks. There was associated cough and difficulty in breathing. A failure to attain expected developmental milestone for his age was elicited. The patient's height was $68 \mathrm{~cm}$, occipito-frontal circumference (OFC) was $42 \mathrm{~cm}$, mid-upper arm circumference (MUAC) was $12 \mathrm{~cm}$ and weighed $5.7 \mathrm{~kg}$ with a history of failure to thrive since early infancy. The patient's prenatal and neonatal histories were significant for birth asphyxia. He was febrile with axillary temperature of 38.5C, moderately dehydrated, tachypneic and tachycardic with irregular pulse and gallop rhythm. The abdomen was mildly distended with visible peristalsis but no palpable mass. The perineum was clear with empty rectum. Neurological examination revealed truncal hypotonia and mild spasticity of the limbs with persistent palmar reflex. Deep tendon reflex mildly exaggerated with motor coordination deficits. The neurodevelopmental assessment was done using the Bayley Scales of Infant and Toddler Development, third edition (Bayley-III), providing a cognitive composite score (CCS), a language composite score (CLS) and a motor composite score (MCS) with a mean of 100 (Standard Deviation of 15). For all subscores, values below normative mean were obtained. An abdominal ultrasonography suggested a bowel obstruction to rule out Hirschsprung's disease. He had a total leucocyte count of $24,550 / \mathrm{mm} 3$, hemoglobin of 9.5 $\mathrm{g} / \mathrm{dL}$, normal blood urea and serum creatinine but low serum sodium and chloride ions of $124 \mathrm{mEq} / \mathrm{L}$ and $85 \mathrm{mEq} / \mathrm{L}$ respectively. Due to the persistent bilious vomiting and ultrasonographic findings, a barium meal study was performed that suggested malrotation and dextrocardia as shown in figure 1 below with associated volvulus and centrally placed liver as shown in figure2 below. The early treatment patient received was broad spectrum antibiotics, cautious correction of the fluid and electrolyte imbalance and zinc 
supplementation. Few hours to the surgery, patient became dyspneic with barely recordable pulses for which several cycles of high quality cardiopulmonary resuscitations (CPRs) were carried out for over $30 \mathrm{~min}$, but could not be resuscitated and unfortunately died.

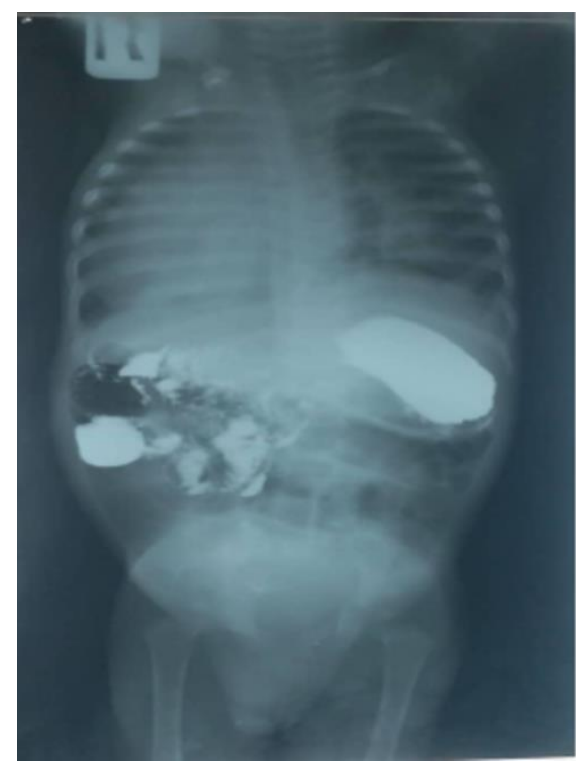

Figure 1. showing dextrocardia, intestinal malrotation and obstruction.

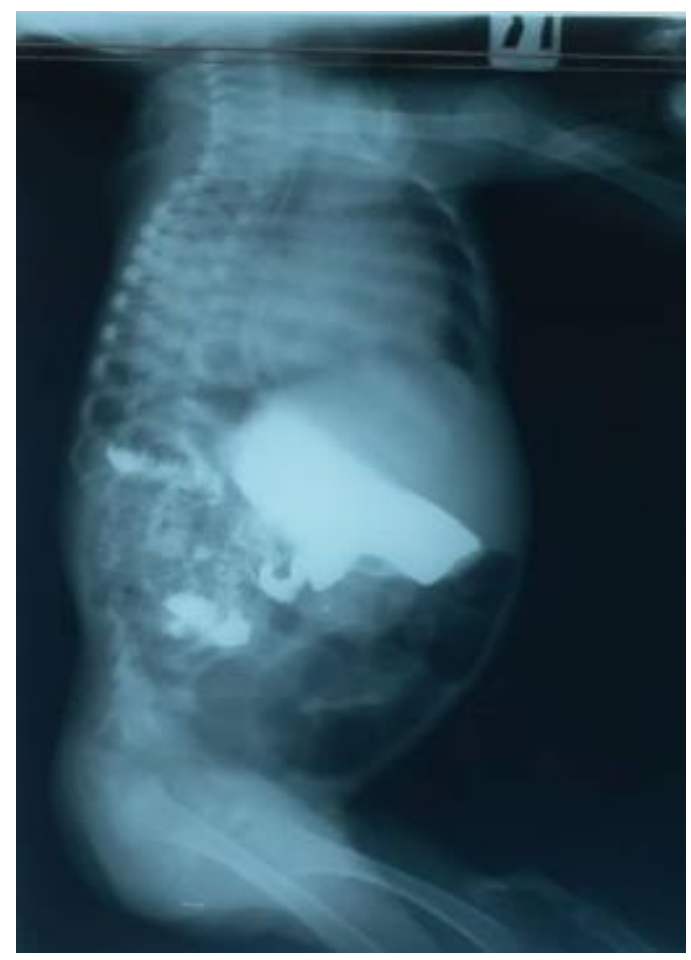

Figure 2. Showing volvulus and centrally placed liver.

\section{Literature Review}

Heterotaxy syndrome is a rare disease characterized by abnormal lateralization of thoraco-abdominal organs across the left-right axis of the body, and usually associated with complex congenital heart disease(CHD) [8]. It is a form of congenital disorders 
manifesting as an abnormal arrangement of thoraco-abdominal organs across the left-right axis of the body due to failure of establishing the normal asymmetry during embryonic development which can be either right atrial isomerism (RAI) or left atrial isomerism (LAI) characterized by bilateral right or left-sidedness of the organs, respectively[9]. The underlying cause of heterotaxy syndrome in majority of cases is unknown, however, cellular and molecular mechanisms were shown to influence the development of the lateralization defects in some cases during early embryogenesis [10]. Very early during gestation, asymmetry signaling is activated by leftward "nodal flow" caused by the unidirectional rotation of monocilia creating and transmitting signals to the left lateral plate mesoderm, where they upregulate the expression of a series of left determinants, such as Nodal, left-right determination factor 2 (Lefty2), and paired-like homeodomain 2 (Pitx2) which in turn stimulate genetic programs in the left side of the body that lead to asymmetric organ morphogenesis (11). Mutations in several genes like CFC1, NODAL, ACVR2B, LEFTY2, GDF1, ZIC3, CRELD1, and NKX2.5 have been implicated in human heterotaxy syndrome more especially when interacted with environmental factors $[10,11,12,13]$.

Although patients with Heterotaxy syndrome may present with normal spleen, but it is traditionally classified into three subtypes based on the characteristic morphology of the spleen as asplenia, single-right-spleen, and polysplenia [14]. The asplenia subtype typically constitutes the right-sided isomerism in which organs normally located on the right side of the body are also found on the left side occurring bilaterally with absence of the left sided organs [5]. The single-right-sided spleen subtype is very rare with extremely few reported studies and therefore no detailed description of it could be found [15]. The polysplenia sub-type typically forms the left-sided isomerism and is usually associated with bilateral bilobed lungs and hyparterial bronchi. It is a condition where organs that are normally present on the left side of the body are present bilaterally with an absence of the right sided organs [11]. Due to the rarity of the disease and improvement in early and rapid diagnosis, the epidemiologic data would be difficult to establish and therefore, the incidence and prevalence might be potentially underestimated [4]. The worldwide prevalence was estimated as slightly more than 1 per 10,000, ranging between 0.88 and 1.7 per 10,000 with estimated prevalence in the United States, Canada and Paris as 1.1 per $10,000,1.3$ per 10,000 and 1.2 per 10,000 respectively. A slightly higher prevalence was reported in parts of Asia with virtually no reliable epidemiological data from Africa [8].

Heterotaxy syndrome is a heterogenous disorder associated with complex cardiac and extra-cardiac abnormalities involving lungs, liver, spleen, intestine, central nervous system and other midline defects with $40-70 \%$ of patients demonstrating involvement of various systems $[7,14,15]$. The syndrome is a form of congenital heart disease (CHD), and the most serious of them, which occurs in approximately $0.8 \%$ of children with CHD and has very poor prognosis when associated with complex cardiac lesions $[9,15,16]$. The commonly observed cardiac anomalies in HS syndrome include abnormal cardiac position like dextrocardia, mesocardia or levocardia; abnormal atrial appendages morphology; abnormal vessel orientation; septal defects; absence of coronary sinus and cardiac rhythm abnormalities [19]. Intestinal rotation abnormality (IRA) is very common in patient with HS which occur as a result of gut rotational defect due to failure of normal counterclockwise rotation of mid gut by $270^{\circ}$, and approximately $40 \%-90 \%$ of children with HS have an IRA. It is a spectrum of disease that typically present with absent bowel fixation by the mesenteric bands thereby predisposing patients to the risks of bowel obstruction, acute or chronic volvulus, and bowel necrosis or infarction $[17,18]$. Therefore, it is necessary to always rule out gut malrotation in HS through abdominal ultrasound or barium examination [17].

Neurological abnormalities are not adequately reported in children with HS, however, some central nervous system (CNS) anomalies like hydrocephalus, absent corpus 
callosum, holonprosencephaly, meningomyelocoele and cerebral dysgenesis were reported and these conditions may give rise to CP [21]. Additionally, panhypopituitarism, absent pituitary infundibulum and ectopic neurohypophysis were also reported in literature [22]. In a study by Ticho and colleagues, midline central nervous system defects like meningomyelocele, porencephalic cyst, cerebellar agenesis, encephalocele, DandyWalker cyst, holoprosencephaly, diplomyelia, and hydromyelia were found in some patients with HS with some of them manifesting clinically as CP [23]. As neural crest cells and their migration during embryonic life play an important role in the development of the heart, neuronal migration abnormalities may lead to serious cerebral malformations and cardiac defects that may present clinically as CP and/or CHD. On the other hand, CHD may predispose fetus to cerebral impairment due to disturbance of cerebral blood flow that may present clinically as $\mathrm{CP}[24]$.

\section{Discussion}

The normal orientation of the organs in the human body is determined early in the embryonic development which is based on genetic information. Loss of such orderly and normal arrangement may result in a disordered and variable arrangement. HS is a complex disorder that occurs as a result of abnormal arrangement of organs and vessels in the chest or abdomen and clinically present as three subgroups as described earlier. The incidence is approximately $1: 10,000$ births and is slightly more prevalent in males than females [25]. In more than half of patients with HS, there is associated abnormal symmetry of liver, abnormally short or truncated pancreas, cardiac and extra-cardiac abnormalities and malrotation $(70-100 \%)$ with or without midgut volvulus, and congenital heart disease was recognized as the major cause of mortality [26]. Our case is a unique case of heterotaxy as the patient presented with dextrocardia, left-sided stomach, centrally placed liver, malrotation, volvulus, features of sepsis suggesting possibility of hyposplenia or asplenia and features of cerebral palsy.

The presence of dextrocardia, left-sided stomach, centrally placed liver, malrotation and volvulus is not uncommon in literatures, however, making diagnosis of malrotation and volvulus in a child with HS is a great challenge to both physicians and radiologists. Additionally, sepsis is very common in children with HS, as in our index case, usually due to hyposplenia or asplenia associated with the condition. Prendiville and colleagues reported an incidence of sepsis in heterotaxy to be $24 \%$ indicating high occurrence of sepsis in HS [27]. In contrary, presence of abnormal neurodevelopmental features and the diagnosis of cerebral palsy are unusual in this case and have not been reported commonly in literature. However, congenital abnormalities like congenital heart disease, congenital atresia of oesophagus and intestine are not unusual in children with cerebral palsy [24]. Therefore, this case report is very important clinically as it discusses a rare occurrence of cerebral palsy in association with HS and would reshape the way clinicians look at HS by considering the possibility of occurrence of neurodevelopmental abnormalities like $\mathrm{CP}$ in children with HS which would have a profound effect on the patient management, especially among survivors. The report has some limitations including lack of other supportive investigative techniques like MRI, CT abdomen, CT angiogram of the chest and Echocardiography.

\section{Conclusion}

Heterotaxy Syndrome is a serious congenital abnormality that has high morbidity and mortality especially among children less than one year of age. In this study, we report an unusual case of cardiac and extracardiac abnormalities in patient with HS with associated cerebral palsy. We have reviewed previous literatures and discuss the etiology and risk factors, incidence and prevalence, clinical presentation, classifications and associated co-morbidities of HS such as congenital heart disease, intestinal rotation abnor- 
malities with or without obstruction, malrotation or volvulus or neurological involvement. These features were virtually observed in our index case. Therefore, a high index of suspicion of cerebral palsy should be considered early in any suspected or confirmed case of HS.

\section{References}

[1] Colver A, Fairhurst C, Pharoah POD. Cerebral palsy. Lancet [Internet]. Elsevier Ltd; 2014;383(9924):1240-9. Available from: http://dx.doi.org/10.1016/S0140-6736(13)61835-8

[2] Medicine D, Rosenbaum PL, Jacobsson B. Proposed definition and classification of cerebral palsy, April 2005 - Introduction Proposed definition and classification of cerebral palsy, April 2005 Martin Bax; Murray Goldstein ; Peter Rosenbaum ; Alan Leviton; et al. 2005; (October 2017).

[3] Cohen MS, Anderson RH, Cohen MI, Atz AM, Fogel M, et al. Controversies, genetics, diagnostic assessment, and outcomes relating to the heterotaxy syndrome. Cardiology in the Young. 2007; 17 (2): 29-43.

[4] Kim, Soo-Jin. Heterotaxy syndrome. Korean circulation journal, 2011. 41(5): 227-232.

[5] Bartram U, Wirbelauer J, Speer CP. Heterotaxy Syndrome - Asplenia and Polysplenia as Indicators of Visceral Malposition and Complex Congenital Heart Disease. Neonatology. 2005; 88(4): 278-290.

[6] Loomba RS, Morales DLS, Redington A. Heterotaxy. In Critical Heart Disease in Infants and Children. 2019; 796-803.

[7] Jacobs JP, Anderson RH, Weinberg PM, Walters HL, Tchervenkov CI, Del Duca D, et al. The nomenclature, definition and classification of cardiac structures in the setting of heterotaxy. Cardiology in the Young. 2007; 17(SUPPL. 2): 1-28.

[8] Lopez KN, Marengo LK, Canfield MA, Belmont JW, Dickerson HA. Racial Disparities in Heterotaxy Syndrome. Birth Defects Research Part A: Clinical and Molecular Teratology. 2015; 103(11): 941-950.

[9] Baban A, Cantarutti N, Adorisio R, Lombardi R, Calcagni G, Piano E, et al. Long-term survival and phenotypic spectrum in heterotaxy syndrome: A 25-year follow-up experience. Int J Cardiol [Internet]. Elsevier B.V.. 2018; 268: 100-105. Available from: https://doi.org/10.1016/j.ijcard.2018.02.050

[10] Liu C, Cao R, Xu Y, Li T, Li F, Chen S, et al. Rare copy number variants analysis identifies novel candidate genes in heterotaxy syndrome patients with congenital heart defects. Genome Medicine. 2018; 1-13.

[11] Shiraishi I, Ichikawa H, Syndrome H, Determination L. Human Heterotaxy Syndrome-from molecular genetics to clinical features, management, and prognosis-." Circulation Journal (2012): CJ-12.

[12] Bamford RN, Roessler E, Burdine RD, Şaplakoğlu U, Dela Cruz J, Splitt M, et al. Loss-of-function mutations in the EGF-CFC gene CFC1 are associated with human left-right laterality defects. Nat Genet. 2000; 26(3): 365-369.

[13] Kosaki R, Gebbia M, Kosaki K, Lewin M, Bowers P, Towbin JA, et al. Left-right axis malformations associated with mutations in ACVR2B, the gene for human activin receptor type IIB. Am J Med Genet. 1999; 82(1): 70-76.

[14] Luo H, Tadepalli G, Mahmad A, Niemiera M, Mathew T. A Rare Case of Heterotaxy Syndrome : Eisenmenger Syndrome with Dextrocardia in an Adult. Journal of Advances in Medicine and Medical Research. 2015; 5(3): 409-413.

[15] Jo DS, Jung SS, Joo CU. A Case of Unusual Visceral Heterotaxy Syndrome with Isolated Levocardia. Korean circulation journal. 2013; 705-709.

[16] Wang X, Shi Y, Zeng S, Zhou J, Zhou J, Yuan H, et al. Comparing levocardia and dextrocardia in fetuses with heterotaxy syndrome : prenatal features, clinical significance and outcomes. BMC Pregnancy and Childbirth. 2017; 1-9.

[17] Mahmoud LA Bin. Rare Extracardiac Anomalies Presented with Right Heterotaxy Syndrome in a Newborn Baby: A Case Report. The American Journal of Case Reports. 2020; 1-5.

[18] Mcgovern E, Kelleher E, Potts JE, Brien JO, Walsh K, Nolke L, et al. Predictors of poor outcome among children with heterotaxy syndrome : a retrospective review. Open heart. 2016; $1-7$.

[19] Mishra S. Cardiac and Non-Cardiac Abnormalities in Heterotaxy Syndrome. The Indian Journal of Pediatrics. (2015): 1135-1146

[20] Pockett CR, Dicken BJ, Rebeyka IM, Ross DB, Ryerson LM. Heterotaxy syndrome and intestinal rotation abnormalities : A survey of institutional practice. Journal of pediatric surgery. 2013; 2078-2083.

[21] Kothari SS. Non-cardiac issues in patients with heterotaxy syndrome. Annals of pediatric cardiology. 2014; 7(3): 187.

[22] Omer A, Haddad D, Pisinski L, Krauthamer A V. The Missing Link: A Case of Absent Pituitary Infundibulum and Ectopic Neurohypophysis in a Pediatric Patient with Heterotaxy Syndrome. Journal of radiology case reports. 2017; 28-34.

[23] Ticho BS, Goldstein AM, Van Praagh R. Extracardiac anomalies in the heterotaxy syndromes with focus on anomalies of midline-associated structures. Am J Cardiol. 2000; 85(6): 729-34.

[24] Pharoah POD. Prevalence and pathogenesis of congenital anomalies in cerebral palsy. Archives of Disease in Childhood-Fetal and Neonatal Edition. 2007; 92(6): F489-F493.

[25] Carneiro DS, Arantes JH, Souza GV, et al. Heterotaxy syndrome: a case report. Radiologia Brasilera. 2013; 46(3): 181-183.

[26] 2Mahalik SK, Khanna S, Menon P. Case Report Malrotation and volvulus associated with heterotaxy syndrome. Journal of Indian Association of Pediatric Surgeons. 2012; 17(3):138-141.

[27] Prendiville TW, Barton LL, Holmes KW. Heterotaxy Syndrome : Defining Contemporary Disease Trends. Pediatric cardiology. 2010; 1052-8. 